\title{
Oral Administration of Ren-Shen-Yang-Rong-Tang 'Ninjin'yoeito' Protects Against Hematotoxicity and Induces Immature Erythroid Progenitor Cells in 5-Fluorouracil-induced Anemia
}

\author{
Fumihide Takano', Yasuyuki Ohta ${ }^{1}$, Tomoaki Tanaka', Kenroh Sasaki ${ }^{2}$, \\ Kyoko Kobayashi ${ }^{2}$, Tomoya Takahashi ${ }^{1}$, Nobuo Yahagi ${ }^{1}$, Fumihiko Yoshizaki ${ }^{2}$, \\ Shinji Fushiya ${ }^{3}$ and Tomihisa Ohta ${ }^{1}$
}

\begin{abstract}
${ }^{1}$ Department of Pharmacognosy and Chemistry of Natural Products, Graduate School of Natural Science and Technology, Kanazawa University, Kanazawa, ${ }^{2}$ 2nd Department of Pharmacognosy, Tohoku Pharmaceutical University, Sendai and ${ }^{3}$ Department of Kampo Pharmaceutical Sciences, Nihon Pharmaceutical University, Saitama, Japan
\end{abstract}

\begin{abstract}
The purpose of this study was to investigate the efficacy of four different Japanese and Chinese herbal prescriptions, Ren-Shen-Yang-Rong-Tang (Ninjin'yoeito, NYT), Chai-Hu-Gui-ZhiGan-Jiang-Tang (Saikokeishikankyoto, SKKT), Si-Jun-Zi-Tang (Shikunshito, SKT) and Si-Wu-Tang (Shimotsuto, SMT), which are traditionally used for anemia and fatigue, against hematotoxicity in mice treated with 5-fluorouracil (5-FU). NYT $1-100 \mathrm{mg} \mathrm{kg}^{-1} \mathrm{day}^{-1}$ injected orally for 7 consecutive days before and after 5-FU injection significantly suppressed reductions in red blood cell, white blood cell and platelet counts in peripheral blood, and accelerated their recovery. Administration of SKKT also produced a slight but significant improvement in 5-FU-induced erythrocytopenia, whereas SMT and SKT could not prevent anemia. Oral injection of NYT also inhibited 5-FU-induced decreases in peripheral reticulocyte and bone marrow cell counts on day 10 , and markedly hastened their recovery on day 20 , in a dosedependent manner. Erythroid progenitor colonies, such as colony forming units-erythroid and burst forming units-erythroid, formed by marrow cells from mice treated with 5-FU were significantly increased by oral administration of NYT. These findings suggest that NYT has the potential to protect against hematotoxicity, and also has hematopoietic activity, through stimulation of immature erythroid progenitor cell differentiation.
\end{abstract}

Keywords: anemia - erythroid progenitor cells - 5-fluorouracil - Kampo medicines Ninjin'yoeito

\section{Introduction}

Severe anemia that is resistant to medical treatment is often observed in patients with malignancies who are undergoing chemotherapy, and pathogenesis of this anemia is multifactorial (1). A low level of circulating

For reprints and all correspondence: Fumihide Takano, Department of Pharmacognosy and Chemistry of Natural Products, Graduate School of Natural Science and Technology, Kanazawa University,

Kakuma-machi, Kanazawa 920-1192, Japan. Tel: +81-76-234-4470;

Fax: +81-76-264-6241; E-mail: takano@p.kanazawa-u.ac.jp hematopoietic growth factor such as erythropoietin (EPO), shortened survival time of circulating red blood cells (RBCs), and a decrease in the number of immature erythroid cells in bone marrow, probably due to chemotherapy or chronic inflammation, have been demonstrated as causes of anemia (2-5). To cope with chronic anemia, blood transfusion, as well as EPO, have been used clinically in various situations. Clinical trials of EPO in patients undergoing cancer chemotherapy have shown prevention of the development of anemia, 
although complete recovery has not been found (6). Clibon et al. (7) have reported that EPO cannot sufficiently overcome the reduced hematopoiesis induced by inflammatory cytokine tumor necrosis factor (TNF)- $\alpha$. Besides hematopoietic growth factors and transfusion, Japanese herbal medicines, such as Juzen-taiho-to, have been used clinically to ameliorate the erythrocytopenia, fatigue or anorexia of patients who are undergoing cancer chemotherapy $(8,9)$. In one clinical trial, Juzentaiho-to has been reported to enhance peripheral blood counts in cancer patients receiving radiation therapy (8), and the active constituents have been identified as oleic and linoleic acids (9). Recently, an anti-cancer polysaccharide, lentinan, has been shown to stimulate proliferation of immature erythroid progenitors, burst forming units-erythroid (BFU-E), and to improve 5-fluorouracil (5-FU)-induced reduction of the number of BFU-E in mice (10). We have also reported that hot-water extracts from Angelica acutiloba Kitagawa, which is used as herbal medicine for postmenstrual blood loss and EPOresistant anemia in chronic renal failure, and its main active polysaccharide constituent increase peripheral $\mathrm{RBC}$ and reticulocyte counts, as well as BFU-E mix and colony forming unit-erythroids (CFU-E) in cultured marrow cells from mice with 5-FU-induced anemia, and these mechanisms are, in part, due to inhibition of inflammatory cytokine production without EPO expression (11). These results indicate that herbal medicines and plant metabolites that stimulate the proliferation of erythroid progenitors, and that have the potential to recover erythrocytopenia in animal models of anemia, may be useful for ameliorating anemia in clinical trials.

In this experiment, we chose four different kinds of Chinese and Japanese herbal medicines: Ren-Shen-YangRong-Tang ('Ninjin'yoeito' in Japanese, NYT), Chai-HuGui-Zhi-Gan-Jiang-Tang (Saikokeishikankyoto, SKKT), Si-Jun-Zi-Tang (Shikunshito, SKT) and Si-Wu-Tang (Shimotsuto, SMT), which are used clinically for postmenstrual blood loss and EPO-resistant anemia, and investigated whether their crude extracts recovered anemia induced by 5-FU in mice. 5-FU exerts its cytotoxic effect mainly by inhibiting thymidylate synthase activity, and this agent has often been used in chemotherapeutic combinations for malignancy. Using a well-established experimental model of 5-FU-induced anemia (10-13), we attempted to compare the therapeutic efficacy of NYT, SMT, SKT and SKKT, and then investigated the pharmacological mechanisms of the effective extracts leading to erythropoiesis.

\section{Methods}

\section{Preparation of Kampo Medicines and Hot-Water Extracts}

Chopped crude drugs, which were standardized by the Japanese Pharmacopoeia (JP) XV, were purchased from
Nakai-kohshindo (Kobe, Japan). Four different Kampo formulas were prepared according to prescriptions for a 1-day dose (14), and are described later (see also Table 1). Each prescription (1-day dose) was decocted in a beaker with $600 \mathrm{ml}$ water by boiling for $30 \mathrm{~min}$ over an electric heater $(600 \mathrm{~W})$, and filtered through absorbent cotton, followed by concentration in vacuo, and freezedrying. The yields for individual extracts are described subsequently. NYT (yield, $8.04 \mathrm{~g}$ ): Rehmannia root (Rehmannia glutinosa, from China) $4.0 \mathrm{~g}$, Japanese Angelica root (Angelica acutiloba, from Japan) $4.0 \mathrm{~g}$, Atractylodes rhizome (Atractylodes ovata, from China) $4.0 \mathrm{~g}$, Poria sclerotium (Poria cocos, from China) $4.0 \mathrm{~g}$, Cinnamon bark (Cinnamomum cassia, from China) $2.5 \mathrm{~g}$, Peony root (Paeonia lactiflora, from Japan) $2.0 \mathrm{~g}$, Polygala root (Polygala tenuifolia, from China) $2.0 \mathrm{~g}$, Citrus unshiu peel (Citrus unshiu, from Japan) $2.0 \mathrm{~g}$, Astragalus root (Astragalus membranaceus, from China) $2.0 \mathrm{~g}$, Ginseng (Panax ginseng, from China) $3.0 \mathrm{~g}$, Glycyrrhiza (Glycyrrhiza uralensis, or Glycyrrhiza glabra, from China) $1.0 \mathrm{~g}$, Schisandra fruit (Schisandra chinensis, from China) $1.0 \mathrm{~g}$. SKKT (yield, $3.50 \mathrm{~g}$ ): Bupleurum root (Bupleurum falcatum, from China) $6.0 \mathrm{~g}$, Cinnamon bark (Cinnamomum cassia, from China) $3.0 \mathrm{~g}$, Trichosanthes root (Trichosanthes kirilowii, from China) $3.0 \mathrm{~g}$, Scutellaria root (Scutellaria baicalensis, from China) $3.0 \mathrm{~g}$, Oyster Shell (Ostrea gigas, from Japan) $3.0 \mathrm{~g}$, processed ginger (steamed rhizome of Zingiber officinale, from China) $1.0 \mathrm{~g}$, Glycyrrhiza (G. uralensis, or G. glabra, from China) 2.0 g. SKT (yield, $4.54 \mathrm{~g}$ ): Ginseng (root of Panax ginseng, from China) $4.0 \mathrm{~g}$, Atractylodes rhizome (Atractylodes ovata, from China) $4.0 \mathrm{~g}$, Poria sclerotium (Poria cocos, from China) $4.0 \mathrm{~g}$, Ginger (rhizome of Zingiber officinale, from China) $1.0 \mathrm{~g}$, Jujube (fruit of Zizyphus jujuba, from China) $2.0 \mathrm{~g}$, Glycyrrhiza (root of G. uralensis, or G. glabra, from China) 2.0 g. SMT (yield, $7.11 \mathrm{~g}$ ): Japanese Angelica root (Angelica acutiloba, from Japan) $4.0 \mathrm{~g}$, Cnidium Rhizome (Cnidium officinale, from Japan) $4.0 \mathrm{~g}$, Peony root (Paeonia lactiflora, from Japan) $4.0 \mathrm{~g}$, Rehmannia root (Rehmannia glutinosa, from China) $4.0 \mathrm{~g}$.

\section{Analysis of Kampo Extracts by Photodiode Array (PDA)-High Performance Liquid Chromatography (HPLC)}

HPLC analysis of the ingredients of NYT extracts was performed using a previously described method (15), with minor modifications. In brief, lyophilized NYT extracts $(1.0 \mathrm{~g})$ were individually extracted with $100 \mathrm{ml}$ methanol by ultrasonication for $30 \mathrm{~min}$. Supernatants were removed by centrifugation $(450 \mathrm{~g})$ and filtrated through a membrane filter (pore size, $0.45 \mu \mathrm{m}$ ). Then, the filtrate was analyzed by HPLC (Hitachi, Tokyo, Japan) equipped with L-7100 pumps, D-7000 interface, L-7455 PDA detector, L-7200 auto sampler, L-7300 column 
Table 1. Ingredients of four Kampo formulas used clinically for anemia

\begin{tabular}{|c|c|c|c|}
\hline Kampo prescription & Plant name & Part used & Composition $(\mathrm{g})$ \\
\hline NYT & Angelica acutiloba Kitagawa & Root & 4.0 \\
\hline \multirow[t]{9}{*}{ Ren-Shen-Yang-Rong-Tang } & Astragalus membranaceus Fisch. & Root & 4.0 \\
\hline & Cinnamomum cassia Blume.* & Bark & 2.5 \\
\hline & Citrus unshiu Marc. & Peel & 2.0 \\
\hline & Glycyrrhiza uralensis Fisch.* & Root & 1.0 \\
\hline & Panax ginseng C.A. Meyer & Root & 3.0 \\
\hline & Polygala tenuifolia Willd. & Root & 2.0 \\
\hline & Poria cocos (FR.) Wolf. & Sclerotium & 4.0 \\
\hline & Rehmannia glutinosa Lib. var. purpurea Makino & Root & 4.0 \\
\hline & Schisandra chinensis (Turcz.) Baill & Fruit & 1.0 \\
\hline & Ostrea gigas & Shell & 3.0 \\
\hline & Scutellaria baicalensis Georgi. & Root & 3.0 \\
\hline & Trichosanthes kirilowii var. japonica & Root & 3.0 \\
\hline & Zingiber officinale Rocs. & Steamed rhizome & 1.0 \\
\hline SKT & Atractylodes ovata DC. & Rhizome & 4.0 \\
\hline \multirow[t]{4}{*}{ Si-Jun-Zi-Tang } & Glycyrrhiza uralensis Fisch. & Root & 2.0 \\
\hline & Panax ginseng C.A. Meyer & Root & 4.0 \\
\hline & Poria cocos (FR.) Wolf. & Sclerotium & 4.0 \\
\hline & Zingiber officinale Rocs. & Rhizome & 1.0 \\
\hline
\end{tabular}

Composition of crude drug in each prescription indicated as 1-day dosage. Asterisks indicate similarity of ingredients between NYT and SKKT.

oven, and Inertsil ODS- 2 column $(250 \times 4.6 \mathrm{~mm}$ i.d., $5 \mu \mathrm{m}$ particle size; GL Sciences, Tokyo, Japan). The solvents used in this analysis were $0.05 \%(\mathrm{w} / \mathrm{w})$ trifluoroacetic acid and $100 \%$ acetonitrile. For detecting ingredients of crude extracts by HPLC, an initial solvent $(100 \%$ trifluoroacetic acid) was applied for $5 \mathrm{~min}$, and then changed to $100 \%$ acetonitrile in a linear gradient manner

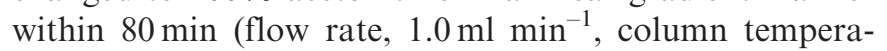
ture $40^{\circ} \mathrm{C}$ ). Effluents were collected and detected by UV at $200-400 \mathrm{~nm}$. Peak assignment was analyzed using system software, D-7000 HSM (Hitachi). Figure 1 shows the results of 3D-HPLC of NYT.

\section{Mice}

The animals were treated according to the 'Guiding Principles for the Care and Use of Animals in the Field' of The Physiological Society of Japan, and the present experiment was approved by the Institute for Experimental Animals, Kanazawa University Advanced Science Research Center, Japan. Female C57BL/6J mice, 8-9 weeks of age, were purchased from Japan SLC (Shizuoka, Japan). Mice were housed in groups of eight in plastic cages with a $12 / 12 \mathrm{~h}$ light/dark cycle and free access to water and mouse chow ad libitum. An adaptation period of at least 1 week for these conditions was allowed before the experiment.

\section{Experimental Protocol}

5-FU (Hoffman La Roche-Kyowa, Tokyo, Japan) at $150 \mathrm{mg} \mathrm{kg}^{-1}\left(0.01 \mathrm{ml} \mathrm{g}^{-1}\right.$ body weight $)$ was dissolved in saline and injected intravenously into the tail vein (on day 0) (11). All samples dissolved in distilled water were injected orally at $1-100 \mathrm{mg} \mathrm{kg}^{-1} \mathrm{day}^{-1}$ once a day for 7 consecutive days (days -3 to +3 ). For the second 


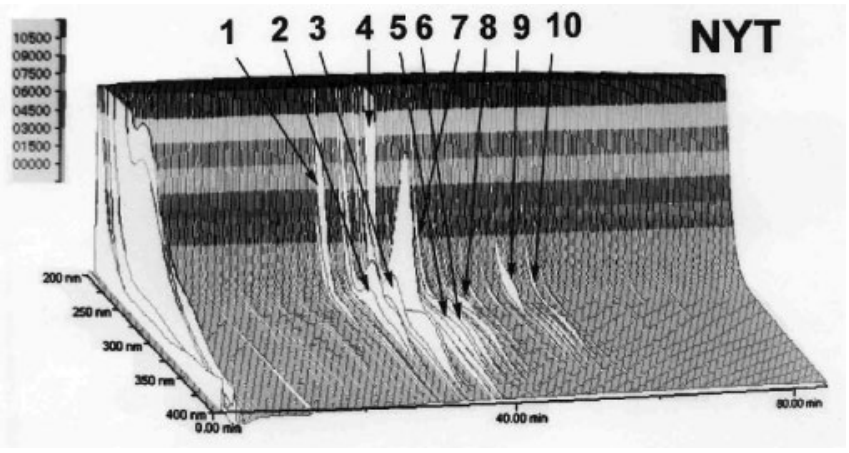

Figure 1. PDA-HPLC profile of NYT extracts. Major peaks identified are: 1, paeoniflorin; 2, liquiritin; 3, narirutun; 4, hesperidin; 5, isoliquiritin apioside; 6, isoliquiritin; 7, liquiritigenin; 8, glycyrrhizin; 9 , cinnamaldehyde; 10 , schizandrin.

experiment, the mice treated with 5-FU were subcutaneously injected with $50 \mathrm{ng}$ murine recombinant interleukin-3 [IL-3; dissolved in pyrogen-free PBS containing $0.5 \%$ bovine serum albumin (BSA); Genzyme, Boston, MA, USA], simultaneously with $5 \mathrm{U}$ murine recombinant EPO (mrEPO; dissolved in pyrogen-free PBS containing $0.5 \%$ BSA; Roche, Mannheim, Germany). The disease control group was treated with vehicle.

\section{Hematological Analysis}

Peripheral blood samples were collected on days 0,5 , 10,15 and 20 by the ocular sinus sampling method with an EDTA-coated capillary tube. White blood cells (WBCs), red blood cells (RBCs), platelets and erythrocytic parameters, including hemoglobin $(\mathrm{Hb})$ concentration and hematocrit $(\mathrm{Ht})$, were counted using an automatic hemocytometer (Horiba, MICROS abc, LC-152, Kyoto, Japan) $(11,16)$. Peripheral reticulocytes were enumerated according to a method described by Brecher and Schneiderman (17). That is, freshly prepared methylene blue solution was mixed with an equal volume of peripheral blood and incubated for $15 \mathrm{~min}$ at room temperature. Then, blood smears were prepared on glass slides using a cytocentrifuge (Hitachi, Ibaraki, Japan) and stained with Wright solution (Muto Pure Chemicals, Tokyo, Japan). Microscopic observation was performed using an ocular micrometer disk, as described by Brecher and Schneiderman (17).

\section{Marrow Cell Preparation and CFU Assay}

Bone marrow was obtained from tibiae and femurs, as described previously (16). Briefly, a cell suspension collected by aspirating the bone with a syringe filled with PBS was washed twice with the same buffer. Marrow cells were counted individually by a hemocytometer, and stored in plastic tubes, on ice, before CFU assays were performed. The marrow cell suspension was then washed twice with $\alpha$-MEM (Invitrogen, Carlsbad,
CA, USA) with $10 \%$ fetal bovine serum (FBS), and resuspended in the same medium. CFU-E and BFU-E in femoral bone marrow were analyzed using methylcellulose-based colony assays $(11,12,18)$. Cell suspensions were gently mixed with $0.8 \%$ methyl cellulose in $\alpha$-MEM medium containing 30\% FBS, $1 \%$ BSA, $200 \mu \mathrm{M}$ hemin and $100 \mu \mathrm{M}$ 2-mercapthoethanol. For developing CFU-E (late erythroid progenitors), $1 \times 10^{5}$ cells ml ${ }^{-1}$ were plated in $35 \mathrm{~mm}$ Petri dishes, and stimulated with $2 \mathrm{UmrEPO} \mathrm{ml}^{-1}$ for 2 days. For developing BFU-E mix (myeloid-mixed early erythroid population), the marrow cell suspensions $\left(2 \times 10^{5}\right.$ cells ml $\left.{ }^{-1}\right)$ were plated in the same dishes, and stimulated with $2 \mathrm{U} \mathrm{mrEPO} \mathrm{ml} l^{-1}$ and $5 \mathrm{ng} \mathrm{IL-} 3 \mathrm{ml}^{-1}$ for 9 days. All dishes were incubated at $37^{\circ} \mathrm{C}$ in a humidified $5 \% \quad \mathrm{CO}_{2}$ incubator. After 2 days (for CFU-E) or 9 days (for BFU-E mix), the methylcellulose discs were transferred to glass slides and fixed with glutaraldehyde solution $(2 \% \mathrm{v} /$ $\mathrm{v}$ in PBS). Both colonies (BFU-E mix and CFU-E) were enumerated under a microscope. Only colonies consisting of at least 10 cells were counted.

\section{Statistics}

Results are expressed as the mean $\pm \mathrm{SE}$ of at least three independent experiments. Statistical significance was determined by Dunnett's multiple test after one-way analysis of variance (ANOVA), by comparison with a physiologically normal or a disease control group, and $P<0.05$ was considered significant.

\section{Results}

\section{PDA-HPLC Profile of NYT}

As depicted in Fig. 1, PDA-HPLC analysis revealed that broad peaks of chemical constituents from NYT appeared at various retention times. UV and LC-MS/ MS analyses of reference compounds indicated the presence of the following known constituents of NYT: paeoniflorin (peak 1) from Peony root, liquiritin (peak 2), isoliquiritin apioside (peak 5), isoliquiritin (peak 6), liquiritigenin (peak 7) and glycyrrhizin (peak 8) from Glycyrrhiza, narirutun (peak 3) and hesperidin (peak 4) from Citrus Unshiu peel, cinnamaldehyde (peak 9) from Cinnamon bark, and schizandrin (peak 10) from Schisandra fruit.

\section{Oral Administration of NYT and SKKT Improved Erythrocytopenia in Mice Treated with 5-FU}

We first examined the kinetics of peripheral RBCs after a higher dose of 5-FU. The experimental protocol is shown in Fig. 2. Peripheral anemia appeared 10 days after intravenous injection of $5-\mathrm{FU} 150 \mathrm{mg} \mathrm{kg}^{-1}$. The recovery 


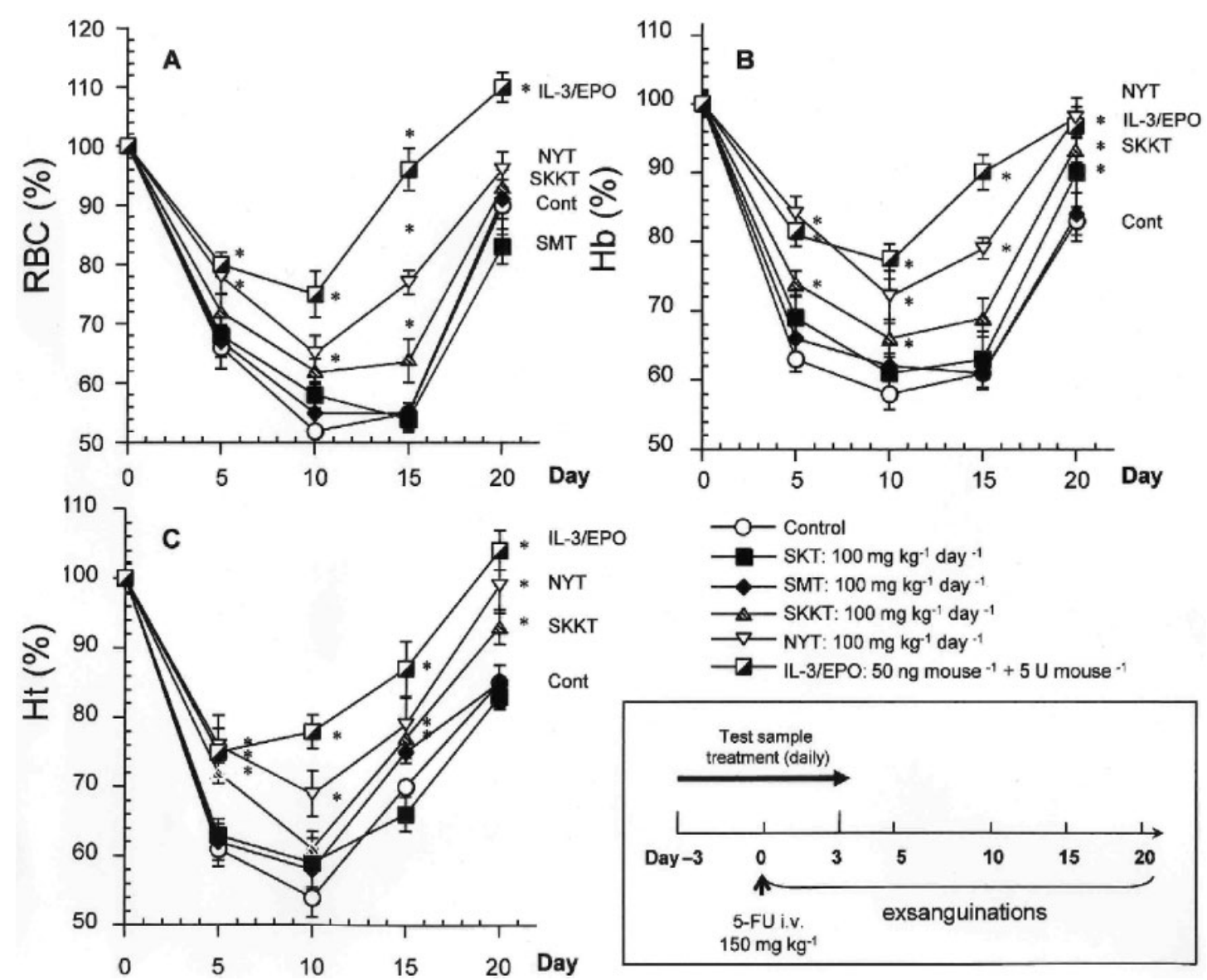

Figure 2. Time course kinetics of $\mathrm{RBCs}, \mathrm{Hb}$, and $\mathrm{Ht}$ in peripheral blood after 5-FU injection. An experimental protocol is also shown. 5-FU $\left(150 \mathrm{mg} \mathrm{kg}^{-1}\right)$ was injected intravenously into mice on day 0 . Peripheral blood samples were collected at the indicated times, and then erythrocyte parameters were measured using an automatic hemocytometer. All tested Kampo samples were orally administered to mice once daily from days -3 to +3 after 5 -FU treatment. One group of 5-FU-treated mice was treated subcutaneously with mrIL-3 (50 ng per mouse) and mrEPO (5 U per mouse). Values are presented as the mean $\pm \mathrm{SE}(n=8-11) .{ }^{*} P<0.05$ compared to 5 -FU-treated mice.

of RBCs, $\mathrm{Ht}$ and $\mathrm{Hb}$ began on day 10 and was almost completed by day 20 (Fig. 2). Oral NYT $100 \mathrm{mg} \mathrm{kg}^{-1}$ day $^{-1}$ for 7 consecutive days significantly improved erythrocytopenia (Fig. 2A) and the reduction in $\mathrm{Ht}$ and $\mathrm{Hb}$ levels on days 10 and 20, whereas SKT and SMT did not improve anemia in mice treated with 5-FU (Fig. 2B and C). SKKT $100 \mathrm{mg} \mathrm{kg}^{-1}$ day $^{-1}$ produced a slight improvement in anemia, although to a lesser extent than NYT treatment. Since IL-3 is known to stimulate differentiation of hematopoietic progenitor cells $(19,20)$, and mrIL-3 has been used as a positive control in previous works $(16,18)$, we tested the efficacy of mrIL-3 (50 ng per mouse) in combination with mrEPO (5 $\mathrm{U}$ per mouse) on 5-FU-induced anemia. On day 10, the 5-FU-induced anemia decreases in $\mathrm{RBCs}, \mathrm{Ht}$; and $\mathrm{Hb}$ were strongly suppressed by subcutaneous injection of $\mathrm{mrIL}-3 / \mathrm{mrEPO}$ for 7 consecutive days (Fig. 2). Furthermore, mrIL-3/ mrEPO hastened recovery of these erythrocyte parameters on day 20 (Fig. 2). Peripheral RBCs, Ht, and Hb levels in physiological control mice were $9.3 \pm 0.2\left(\times 10^{6}\right.$ cells ml $\left.^{-1}\right)$, $41.5 \pm 0.9 \%$, and $11.6 \pm 0.2 \mathrm{~g} \mathrm{dl}^{-1}$, respectively. None of the treatments showed any toxic effects in normal mice, as demonstrated by the fact that body and liver weights did not change (data not shown), and the activity of plasma hepatic marker enzyme, alanine aminotransferase (ALT, EC 2.6.1.2), was not elevated $\left(12.1 \pm 1.0 \mathrm{U}^{-1}\right.$, vehicle once a day for 7 consecutive days, $n=3 ; 18.0 \pm 1.5 \mathrm{Ul}^{-1}$, $100 \mathrm{mg} \mathrm{kg}^{-1}$ day $^{-1}$ NYT once a day for 7 days, $n=5$; $19.9 \pm 2.0 \mathrm{U} \mathrm{l}^{-1}, 100 \mathrm{mg} \mathrm{kg}^{-1} \mathrm{day}^{-1}$ SKKT once a day for 7 days, $16.6 \pm 0.2 \mathrm{U}^{-1}, 100 \mathrm{mg} \mathrm{kg}^{-1} \mathrm{day}^{-1}$ SKT once a day for 7 days, $n=5 ; 15.1 \pm 1.1 \mathrm{U}^{-1}, 100 \mathrm{mg} \mathrm{kg}^{-1}$ day $^{-1}$ SMT once a day for 7 days, $n=5$ ).

\section{NYT Suppressed Leukocytopenia and Thrombocytopenia as Well as Reduction of Peripheral Reticulocyte Counts Induced by 5-FU}

Since oral NYT was more effective than the other Kampo extracts for improving erythrocytopenia, we next examined the dose-response of the protective activity of NYT against hematotoxicity in anemic mice. 5-FU administered to normal mice reduced not only the 
Table 2. Effects of NYT and IL-3 plus EPO on WBC, RBC and platelet counts and hematologic parameters in peripheral blood after 5-FU administration

\begin{tabular}{|c|c|c|c|c|c|c|c|}
\hline Day & Treatment & $\begin{array}{l}\text { Dose } \\
\left(\mathrm{mg} \mathrm{kg}^{-1} \text { per day) }\right.\end{array}$ & $\begin{array}{l}\text { WBC } \\
\left(\times 10^{3} \mu 1^{-1}\right)\end{array}$ & $\begin{array}{l}\mathrm{RBC} \\
\left(\times 10^{6} \mu \mathrm{l}^{-1}\right)\end{array}$ & $\begin{array}{l}\mathrm{Ht} \\
(\%)\end{array}$ & $\begin{array}{l}\mathrm{Hb} \\
\left(\mathrm{g} \mathrm{dl}^{-1}\right)\end{array}$ & $\begin{array}{l}\text { PLT } \\
\left(\times 10^{3} \mu 1^{-1}\right)\end{array}$ \\
\hline \multirow[t]{5}{*}{0} & Normal & - & $3.8 \pm 0.1$ & $9.7 \pm 0.3$ & $44.2 \pm 0.5$ & $14.0 \pm 0.1$ & $338 \pm 6$ \\
\hline & Control & - & $3.3 \pm 0.2$ & $9.5 \pm 0.2$ & $43.8 \pm 1.0$ & $14.9 \pm 0.3$ & $407 \pm 6$ \\
\hline & NYT & 1 & $4.1 \pm 0.3$ & $9.9 \pm 0.1$ & $47.1 \pm 0.2$ & $15.5 \pm 0.1$ & $467 \pm 14$ \\
\hline & NYT & 10 & $5.3 \pm 0.1^{*}$ & $10.2 \pm 0.2$ & $47.1 \pm 0.9$ & $15.7 \pm 0.2$ & $520 \pm 12$ \\
\hline & NYT & 100 & $5.2 \pm 0.1$ & $10.1 \pm 0.1$ & $46.7 \pm 0.4$ & $15.3 \pm 0.1$ & $432 \pm 15$ \\
\hline \multirow[t]{5}{*}{10} & Control & - & $1.9 \pm 0.2^{\#}$ & $6.0 \pm 0.3^{\#}$ & $27.3 \pm 1.2^{\#}$ & $9.5 \pm 0.1^{\#}$ & $126 \pm 9^{\#}$ \\
\hline & NYT & 1 & $2.4 \pm 0.2$ & $6.9 \pm 0.2 *$ & $30.3 \pm 0.3^{*}$ & $10.6 \pm 0.3^{*}$ & $109 \pm 1$ \\
\hline & NYT & 10 & $4.2 \pm 0.9^{*}$ & $7.6 \pm 0.3^{*}$ & $34.0 \pm 1.1^{*}$ & $11.5 \pm 0.4^{*}$ & $137 \pm 8^{*}$ \\
\hline & NYT & 100 & $4.2 \pm 0.4^{*}$ & $8.3 \pm 0.1^{*}$ & $40.4 \pm 0.1^{*}$ & $12.6 \pm 0.2^{*}$ & $146 \pm 8^{*}$ \\
\hline & IL-3/EPO & $50 \mathrm{ng}+5 \mathrm{U}$ & $2.8 \pm 0.4^{\#}$ & $8.1 \pm 0.2^{*}$ & $41.1 \pm 0.2^{*}$ & $13.0 \pm 0.1^{*}$ & $163 \pm 2 *$ \\
\hline \multirow{2}{*}{20} & NYT & 100 & $16.2 \pm 1.7^{*}$ & $9.0 \pm 0.3^{*}$ & $43.5 \pm 0.7$ & $13.5 \pm 0.2$ & $1493 \pm 188$ \\
\hline & IL-3/EPO & $50 \mathrm{ng}+5 \mathrm{U}$ & $13.1 \pm 1.6$ & $10.1 \pm 1.0^{*}$ & $48.2 \pm 0.1^{*}$ & $15.2 \pm 0.1^{*}$ & $1512 \pm 90^{*}$ \\
\hline
\end{tabular}

Mice were injected orally with NYT or subcutaneously with IL-3 (50 ng per mouse) and EPO (5 U per mouse) once daily for 7 consecutive days from 3 days before to 3 days after 5-FU injection. Peripheral blood samples were collected on days 0,10 and 15 , and hematologic parameters were measured by hemocytometer. Data are expressed as the mean $\pm \mathrm{SE}$ of three independent experiments.

${ }^{\#} P<0.05$ compared to normal mice. $* P<0.05$ compared to 5 -FU-treated mice.

erythrocytopenia by $38.1 \%$, but also leukocytopenia and thrombocytopenia by 50.0 and $62.7 \%$ on day 10 , respectively (Table 2 ). These reductions were recovered on day 20, and rebound reactions were observed in the case of platelet and leukocyte counts at this time (Table 2). NYT suppressed 5-FU-induced anemia and accelerated the recovery of erythrocytopenia at doses of $1-100 \mathrm{mg} \mathrm{kg}^{-1} \mathrm{day}^{-1}$ in a dose-dependent manner (Table 2). Furthermore, oral NYT also suppressed leukocytopenia in a dose-dependent manner, but did not affect thrombocytopenia at any dose (Table 2). Subcutaneous injection of mrIL-3/mrEPO, as a positive control, markedly improved anemia and thrombocytopenia induced by 5 -FU.

Because peripheral reticulocyte count reflects erythropoiesis (21), we enumerated peripheral reticulocytes and femoral marrow cells in mice treated with NYT after 5-FU injection. The numbers of myelocytes in bone marrow and reticulocytes in peripheral blood were dramatically reduced 10 days after 5-FU injection (Fig. $3 \mathrm{~A}$ and $\mathrm{C}$ ). On day 20, the number of marrow cell was still reduced (Fig. 3B), whereas reticulocyte count had recovered (Fig. 3D). Bone marrow seems to be more sensitive than peripheral reticulocytes to 5-FU (Fig. 3). NYT $100 \mathrm{mg} \mathrm{kg}^{-1}$ day $^{-1}$ significantly protected against the 5-FU-induced cytotoxicity of reticulocytes and myelocytes, and hastened recovery of the number of these hematopoietic cells (Fig. 3B and D). These effects of
NYT were more pronounced on day 20 than on day 10 . Protective effects against myelotoxicity and hematotoxicity were also observed in mice treated with subcutaneous mrIL-3 and mrEPO (Fig. 3).

\section{NYT-Stimulated Immature Erythroid Colony-Formation in Cultured Marrow Cells}

Since NYT showed the greatest anti-anemic activity, the effect of oral NYT on the expression of immature erythroid progenitor cells obtained from anemic mice was examined. Table 3 shows the results of the colonyforming assay for IL-3/EPO-induced BFU-E mix and EPO-induced CFU-E. The developing colonies of BFU-E mix and CFU-E in cultured bone marrow cells from normal mice were $\sim 3200$ and 44000 per femur, respectively. Intravenous 5-FU dramatically reduced their formation by up to $90 \%$ on day 5 , and only CFU-E formation was completely recovered on day 10 (Table 3). The reduced BFU-E mix was recovered by up to $60 \%$ on day 15 . NYT $1-100 \mathrm{mg} \mathrm{kg}^{-1} \mathrm{day}^{-1}$ increased development of both colonies in a dose-dependent manner, and recovery of BFU-E mix and CFU-E formation was greater in the NYT-treated mice compared with that in the disease control and normal groups (Table 3). A tendency for their colonies to increase was observed in mice treated with NYT, even at a low dose ( $1 \mathrm{mg} \mathrm{kg}^{-1} \mathrm{day}^{-1}$, Table 3). Moreover, subcutaneous 


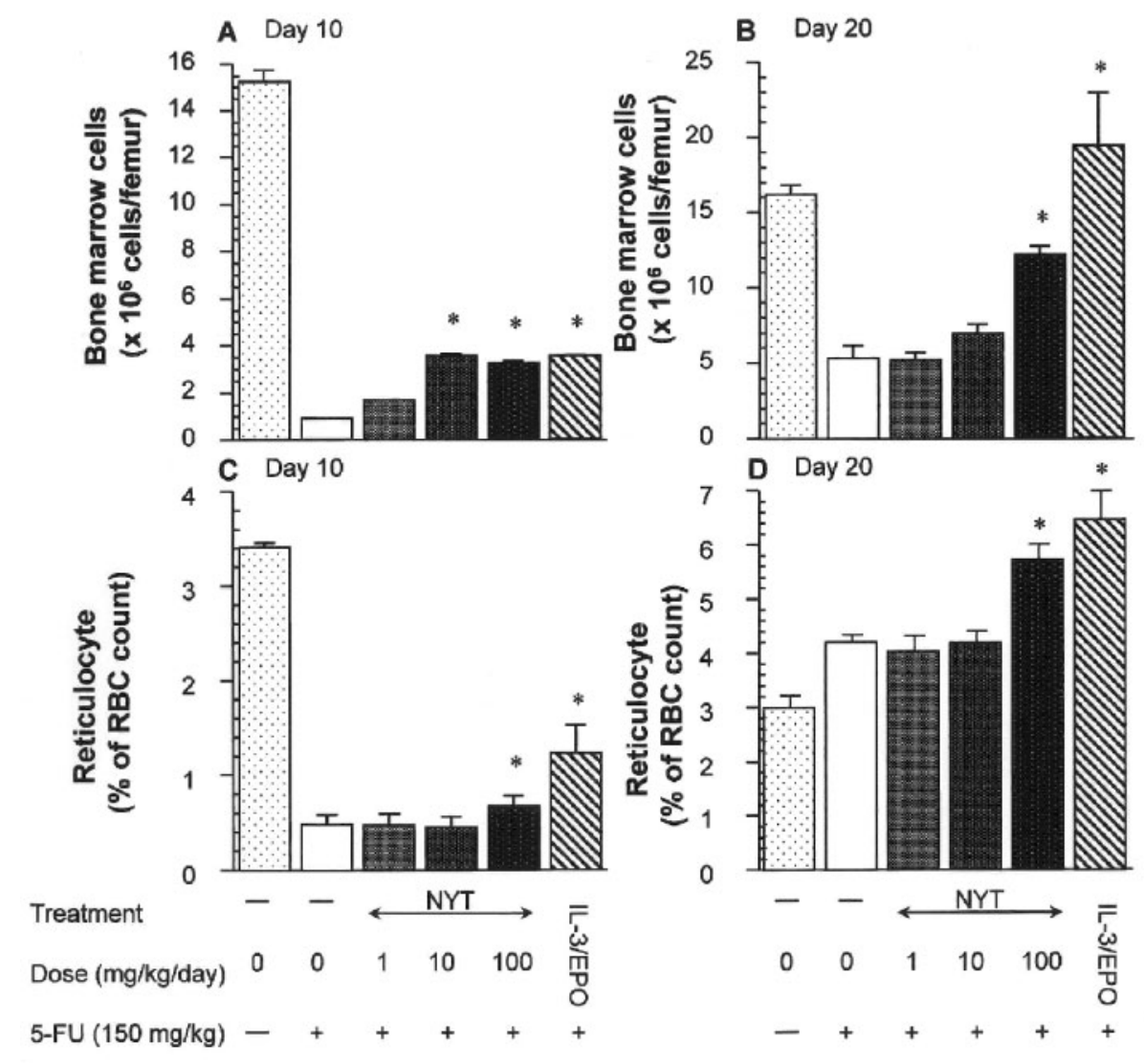

Figure 3. Effect of NYT on the reduced number of bone marrow cells and reticulocytes in mice treated with 5-FU. The number of peripheral reticulocytes and femur marrow cells were determined at 10 and 15 days after 5-FU injection. All tested Kampo samples were administered orally to mice once daily from days -3 to +3 after 5-FU treatment. One group of 5-FU-treated mice was treated subcutaneously with mrIL-3 (50 ng per mouse) and mrEPO (5 $\mathrm{U}$ per mouse). Values are presented as the mean $\pm \mathrm{SE}(n=8-11)$. ${ }^{*} P<0.05$ compared to 5 -FU-treated mice.

mrIL-3 (50 ng per mouse) and mrEPO (5 U per mouse) markedly stimulated colony formation of BFU-E mix and CFU-E (Table 3). Oral administration of NYT (Table 3) and other Kampo extracts (data not shown) did not affect marrow cell viabilities at any time points. Although no data are shown, increased BFU-E mix and CFU-E colony formation in mice-treated with NYT $\left(100 \mathrm{mg} \mathrm{kg}^{-1} \mathrm{day}^{-1}\right.$ ) or mrIL-3/EPO on day 15 (Table 3) reverted to normal levels on day 30 .

\section{Discussion}

To elucidate therapeutic evidences for anti-anemic effect of Kampo medicine, we evaluated the therapeutic effects of four Kampo extracts, traditionally used for anemia and fatigue, on 5-FU-induced anemia. Fujii et al. (22), have previously reported that intraperitoneal injection of NYT $625 \mu \mathrm{g}$ into BALB/c mice significantly accelerates recovery of erythrocyte count after transplantation of bone marrow cells from syngeneic mice, and this regimen also enhances the total number of CFU-E, BFU-E, and CFU-macrophages in bone marrow and spleen after lethal total body irradiation and syngeneic bone marrow transplantation. However, it is well known that intraperitoneal or intravenous injection of test substances contaminated with endotoxin interferes with experimental results in vivo, because endotoxin is widely distributed in natural materials, and causes non-specific immune responses through monocyte activation (23). Indeed, endotoxin reportedly induces hematopoietic growth factors such as granulocyte-macrophage colony stimulating factor (GM-CSF), G-CSF and M-CSF in monocytes $(24,25)$. Therefore, the net efficacy of NYT in anemia is still unknown. In order to avoid the interference of endotoxin and evaluate the net effects of Kampo extracts on anemia, we used endotoxin-non-responder C57BL/6J mice and oral injection in this study.

Of the Kampo extracts tested in this experiment, oral injection of NYT into $\mathrm{C} 57 \mathrm{BL} / 6 \mathrm{~J}$ mice protected against myelotoxicity and hastened recovery of anemia (Fig. 2, Table 2). We found that SKKT also significantly prevented erythrocytopenia caused by 5 -FU, but its effect was weaker than that of NYT (Fig. 2). These results therefore strongly suggest that NYT and SKKT have net anti-anemic activity. Furthermore, the timing of NYT and SKKT administration with anemic patients is 
Table 3. Effects of NYT and IL-3 plus EPO on formation of CFU-E and BFU-E colonies in cultured marrow cells from mice treated with 5-FU

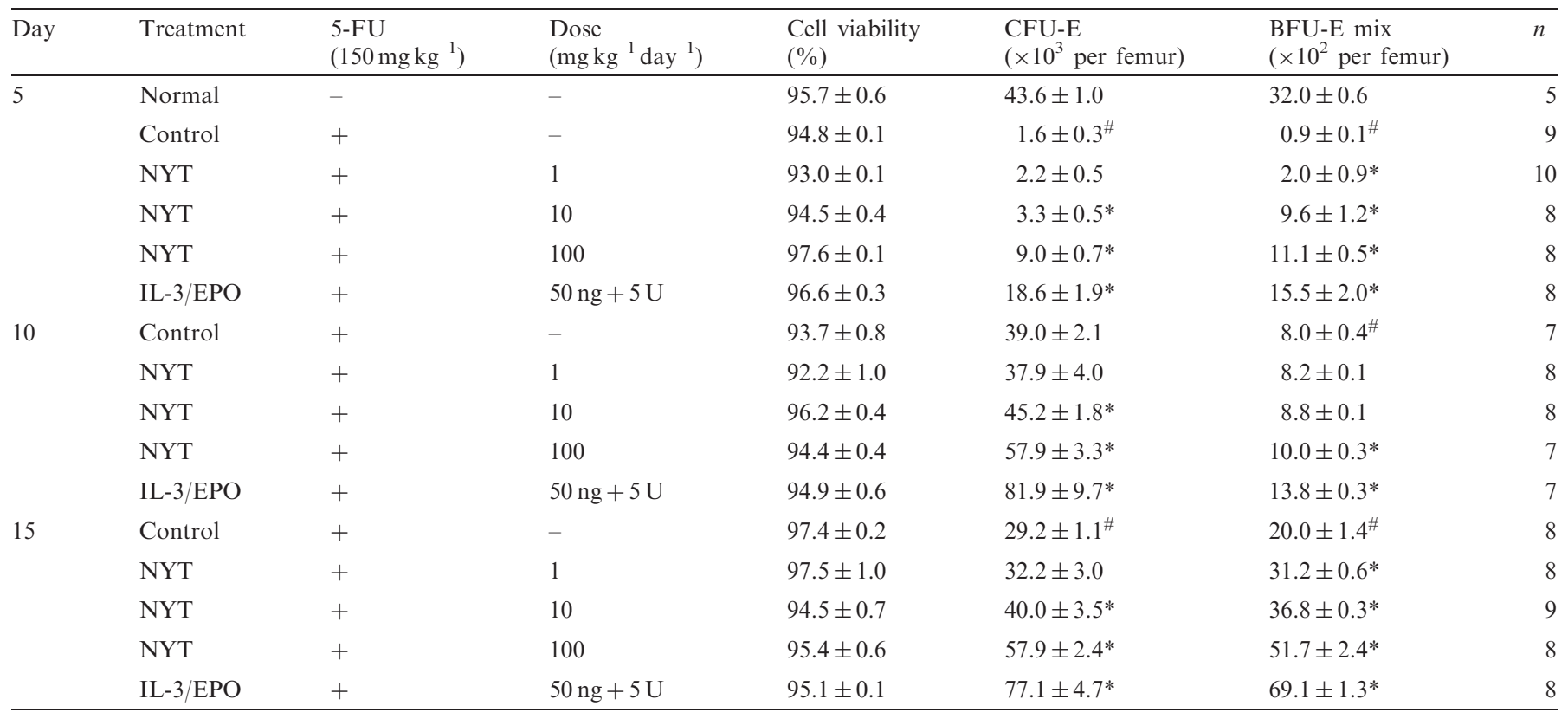

Mice were injected orally with NYT or subcutaneously with IL-3 (50 ng per mouse) and EPO (5 U per mouse) for 7 consecutive days before and after 5-FU injection. Marrow cells were collected at 5, 10 and 15 days after 5-FU injection, and cultured with $2 \mathrm{U} \mathrm{ml}^{-1} \mathrm{EPO}$ with (for BFU-E mix) or without $5 \mathrm{ng} \mathrm{ml}^{-1} \mathrm{IL}-3$ (for CFU-E). The developing colonies were enumerated 2 (CFU-E) or 9 (BFU-E mix) days after cultivation. Data are expressed as the mean $\pm \mathrm{SE}$.

${ }^{\sharp} P<0.05$ compared to normal mice. ${ }^{*} P<0.05$ compared to 5 -FU-treated mice.

clinically different, which may explain why the protective effect of SKKT is weaker than that of NYT (Fig. 2).

We have recently reported that orally administered hot-water extract of Angelica roots (Angelica acutiloba Kitagawa) and an extract fraction rich in polysaccharide protect against erythrocytopenia, and significantly improve recovery from 5-FU-induced anemia (11). Unexpectedly, SMT, which is mainly composed of Angelica roots (Table 1), could not ameliorate 5-FUinduced anemia, even at a higher dose $\left(500 \mathrm{mg} \mathrm{kg}^{-1} \mathrm{day}^{-1}\right.$, data not shown), in contrast to the effect of NYT (also composed of Angelica roots) (Fig. 2). Although it is unclear why SMT could not prevent erythrocytopenia in this experiment, it could be discussed that the other crude drug in the SMT preparation affects the anti-anemic effect of Angelica root (11). Kiyohara et al. (26) recently reported that combined decoction of all essential herbs in Kampo prescription is important to elicit the combination of ingredients required for expression of the specific activity. Hence, we are now planning to investigate the influence of combination of crude Angelica root-containing drugs on anti-anemic activity in the same model, and to isolate further active constituents from hot-water extracts of NYT. Indeed, Juzentaihoto (Shi-Quan-DaBu-Tang in Chinese), whose active substances have been identified as oleic and linoleic acids, is well known to have anti-anemic activity, and to stimulate proliferation of hematopoietic stem cells in vitro and in vivo (9).
NYT has been reported to protect against 5-FU- and cyclophosphamide-induced leukocytopenia at a dose of $1000 \mathrm{mg} \mathrm{kg}^{-1}$ day $^{-1}$ (for 4 consecutive days), when co-administered with G-CSF (27). In this study, identical results were obtained in 5-FU-treated mice treated with oral NYT at $1-100 \mathrm{mg} \mathrm{kg}^{-1} \mathrm{day}^{-1}$; these doses of NYT were sufficient for recovery from 5-FU-induced leukocytopenia, without co-administration of any other hematopoietic growth factor such as GM-CSF (Table 2). Hence, it is clear that NYT itself has the potential to induce recovery from erythrocytopenia and leukocytopenia in anemic mice.

5-FU has been shown not to damage hematopoietic stem cells directly $(28,29)$, because stem cells rapidly begin proliferation after 5-FU chemotherapy and become sensitive to lineage-specific hematopoietic growth factors (30). Intravenous 5-FU caused a decrease in the RBC, $\mathrm{Hb}$ and $\mathrm{Ht}$ levels in mice during the experimental period (20 days), with the maximum decrease noted on day 10 (Fig. 2). Estimation of the immature erythroid cells in peripheral blood and bone marrow demonstrated that there was an increase in the number of those cells on days 10 and 20 (Fig. 3). Since CFU-E is less mature than peripheral reticulocytes, the maximum recovery of CFU-E was on day 10 (Table 2) and the recovery of reticulocytes was on day 20 (Fig. 3D). Furthermore, as BFU-E is known to be more immature than CFU-E, BFU-E recovery was expected to occur earlier than that 
of CFU-E. However, the maximum recovery of BFU-E mix, which contains immature myeloid cells and megakaryocytes, was on day 15 in a CFU assay (Table 3). Our results are in agreement with those of Rich (12), who demonstrated erythropoiesis in mice after 5-FU injection. We found that oral NYT increased the number of marrow cells and reticulocytes, especially on day 20 (Fig. $3 \mathrm{~B}$ and $\mathrm{D}$ ). Furthermore, NYT significantly increased the numbers of both CFU-E and BFU-E mix colonies on days 10 and 15 (Table 3). These results suggest that NYT stimulates early differentiation of erythroid lineage cells in bone marrow after 5-FU toxicity, thereby promoting hematopoiesis.

We have recently reported that the detailed mechanisms by which hot-water extracts of Angelica roots increase recovery of erythrocytopenia, and stimulate differentiation of erythroid progenitors without promoting EPO synthesis, may be partly due to inhibiting the secretion of inflammatory cytokines such as TNF- $\alpha$ and interferon (IFN)- $\gamma$ (11). Besides the therapeutic efficacy of NYT in anemia, it has been reported that NYT suppresses production of TNF- $\alpha$ (31) in cultured rabbit alveolar macrophages, and intraperitoneal NYT in mice also inhibits IFN- $\gamma$ production in splenocytes co-stimulated with anti-CD3 monoclonal antibody (32). Although we did not measure the expression of EPO mRNA in liver and kidney after NYT treatment, it can be assumed that NYT treatment promotes erythropoiesis, possibly in part, due to inflammatory cytokine production (TNF- $\alpha$ and IFN- $\gamma$ ), the presence of which may suppress the differentiation of erythroid progenitor cells. To clarify the detailed anti-anemic mechanism of NYT (and SKKT), further investigations of the effect of NYT on EPO expression and cytokine production in 5-FU-induced anemia are required.

In summary, the present study demonstrates that oral NYT can ameliorate 5-FU-induced anemia and hasten recovery of erythrocytopenia, by stimulating the expression of early erythroid progenitor cells. Therefore, NYT (and SKKT) has therapeutic and prophylactic potential for erythrocytopenia and leukocytopenia, especially when caused by anti-cancer agents.

\section{Acknowledgement}

The authors would like to thank Ms Cui Ming-Yue (MC) of the Graduate School of Natural Science and Technology, Kanazawa University, Japan, for her generous advice.

\section{References}

1. Griggs JJ. Reducing the toxicity of anticancer therapy: new strategies. Leuk Res 1998;22 (Suppl 1): S27-33.

2. DeGowin RL, Gibson DP. Erythropoietin and the anemia of mice bearing extramedullary tumor. J Lab Clin Med 1979;94:303-11.
3. Zucker S, Lysik RM, Friedman S. Diminished bone marrow responsiveness to erythropoietin in myelophthisic anemia. Cancer 1976;37:1308-15.

4. Casadevall N. Cellular mechanism of resistance to erythropoietin. Nephrol Dial Transplant 1995;10 (Suppl 6): 27-30.

5. Means RT Jr, Dessypris EN, Krantz SB. Inhibition of human erythroid colony-forming units by interleukin-1 is mediated by gamma interferon. $J$ Cell Physiol 1992;150:59-64.

6. Lundholm K, Daneryd P, Bosaeus I, Korner U, Lindholm E. Palliative nutritional intervention in addition to cyclooxygenase and erythropoietin treatment for patients with malignant disease: effects on survival, metabolism, and function. Cancer 2004;100:1967-77.

7. Clibon U, Bonewald L, Caro J, Roodman G.D. Erythropoietin fails to reverse the anemia in mice continuously exposed to tumor necrosis factor-alpha in vivo. Exp Hematol 1990;18:438-41.

8. Nabeya K, Ri S. Effects of oriental medical herbs on the restoration of the human body before and after operation. In: Proceedings of the Symposium of Wakan-Yaku. 1983;16:201-8.

9. Hisha H, Yamada H, Sakurai MH, Kiyohara H, Li Y, Yu C, et al. Isolation and identification of hematopoietic stem cell-stimulating substances from Kampo (Japanese herbal) medicine, Juzen-taiho-to. Blood 1997;90:1022-30.

10. Takatsuki F, Miyasaka Y, Kikuchi T, Suzuki M, Hamuro J. Improvement of erythroid toxicity by lentinan and erythropoietin in mice treated with chemotherapeutic agents. Exp Hematol 1996;24:416-22.

11. Hatano R, Takano F, Fushiya S, Michimata M, Tanaka T, Kazama I, et al. Water-soluble extracts from Angelica acutiloba Kitagawa enhance hematopoiesis by activating immature erythroid cells in mice with 5-fluorouracil-induced anemia. Exp Hematol 2004:32:918-24.

12. Rich IN. The effect of 5-fluorouracil on erythropoiesis. Blood 1991;77:1164-70.

13. Weiterova L, Hofer M, Pospisil M, Znojil V, Vacha J, Vacek A, et al. Influence of the joint treatment with granulocyte colonystimulating factor and drugs elevating extracellular adenosine on erythropoietic recovery following 5-fluorouracil-induced haematotoxicity in mice. Eur J Haematol 2000;65:310-6.

14. Otsuka K, Yakazu D. "Keiken Kampo-shohoh-bunryo-shu," ed. by under supervision of Idohno-nihon-sha, Yokosuka, Japan, 1973.

15. Kobayashi J, Seiwa C, Sakai T, Gotoh M, Komatsu Y, Yamamoto M, et al. Effect of a traditional Chinese herbal medicine, Ren-Shen-Yang-Rong-Tang (Japanese name: Ninjin-Youei-To), on oligodendrocyte precursor cells from aged-rat brain. Int Immunopharmacol 2003;3:1027-39.

16. Takano F, Tanaka T, Aoi J, Yahagi N, Fushiya S. Protective effect of (+)-catechin against 5-fluorouracil-induced myelosuppression in mice. Toxicology 2004;201:133-42.

17. Brecher G, Schneiderman M. A time-saving device for the counting of reticulocytes. Am J Clin Pathol 1950;20:1079-83.

18. Takano F, Tanaka T, Tsukamoto E, Yahagi N, Fushiya S. Isolation of (+)-catechin and (-)-epicatechin from Actinidia arguta as bone marrow cell proliferation promoting compounds. Planta Med 2003;69:321-6.

19. Clark SC, Kamen R. The human hematopoietic colony-stimulating factors. Science 1987;236:1229-37.

20. Mangi MH, Newland AC. Interleukin-3 in hematology and oncology: current state of knowledge and future directions. Cytokines Cell Mol Ther 1999;5:87-95.

21. Kakiuchi S, Kobayshi M, Satomi Y, Miura D, Kasahara Y, Kondo S. Flow cytometric analysis of erythropoietic abnormality: changes in the cell maturity index of reticulocytes and retic distribution index are useful as indicators of erythropoietic toxicity in non-clinical studies. $J$ Toxicol Sci 2006;31:111-22.

22. Fujii Y, Imamura M, Han M, Hashino S, Zhu X, Kobayashi H, et al. Recipient-mediated effect of a traditional Chinese herbal medicine, Ren-shen-yang-rong-tang (Japanese name: Ninjin-youeito), on hematopoietic recovery following lethal irradiation and syngeneic bone marrow transplantation. Int J Immunopharmacol 1994;16:615-22.

23. Chong KT, Huston M. Implications of endotoxin contamination in the evaluation of antibodies to lipopolysaccharides in a murine model of gram-negative sepsis. $J$ Infect Dis 1987;156:713-9. 
24. Sieff CA, Niemeyer CM, Faller DV. Human colony-stimulating factors and stromal cell function. Soc Gen Physiol Ser 1988;43:47-55.

25. Burgess AW, Metcalf $\mathrm{D}$. The nature and action of granulocytemacrophage colony stimulating factors. Blood 1980;56:947-58.

26. Kiyohara H, Matsumoto $\mathrm{T}$, Yamada H. Combination effect of herbs in a multi-herbal formula: expression of Juzen-taiho-to's immuno-modulatory activity on the intestinal immune system. Evid Based Complement Alternat Med 2004;1:83-91.

27. Miura S, Takimoto H, Yoshikai Y, Kumazawa Y, Yamada A, Nomoto K. Protective effect of ren-shen-yang-rong-tang (Ninjin-youei-to) in mice with drug-induced leukopenia against Pseudomonas aeruginosa infection. Int $J$ Immunopharmacol 1992;14:1249-57.

28. Lerner C, Harrison DE. 5-Fluorouracil spares hemopoietic stem cells responsible for long-term repopulation. Exp Hematol 1990;18:114-8.
29. Down JD, Ploemacher RE. Transient and permanent engraftment potential of murine hematopoietic stem cell subsets: differential effects of host conditioning with gamma radiation and cytotoxic drugs. Exp Hematol 1993;21:913-21.

30. Harrison DE, Lerner CP. Most primitive hematopoietic stem cells are stimulated to cycle rapidly after treatment with 5-fluorouracil. Blood 1991;78:1237-40.

31. Aoki T, Kojima T, Kameda N, Yoshijima S, Ono A, Kobayashi Y. Anti-inflammatory effect of a traditional Chinese medicine, renshen-yang-rong-tang (Japanese name: ninjin-youei-to), on alveolar macrophages stimulated by RANTES or TNF-alpha. Arerugi 1994:43:663-7.

32. Nakada T, Watanabe K, Jin GB, Triizuk K, Hanawa T. Effect of ninjin-youei-to on Th1/Th2 type cytokine production in different mouse strains. Am J Chin Med 2002;30:215-23.

Received February 27, 2007; accepted June 9, 2007 


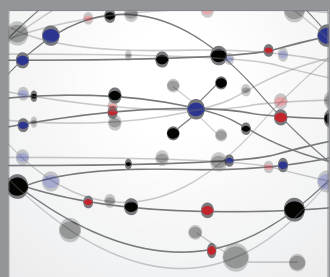

The Scientific World Journal
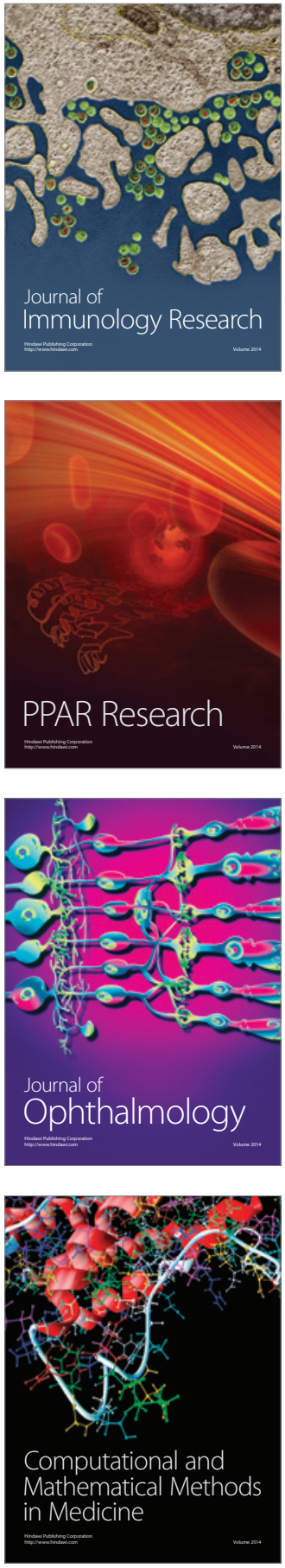

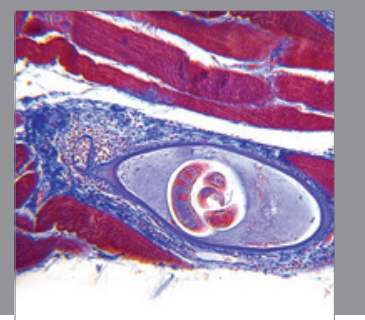

Gastroenterology

Research and Practice
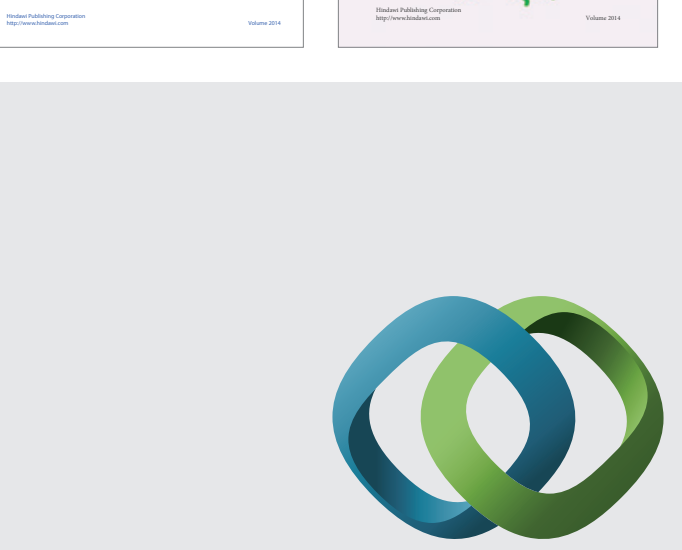

\section{Hindawi}

Submit your manuscripts at

http://www.hindawi.com
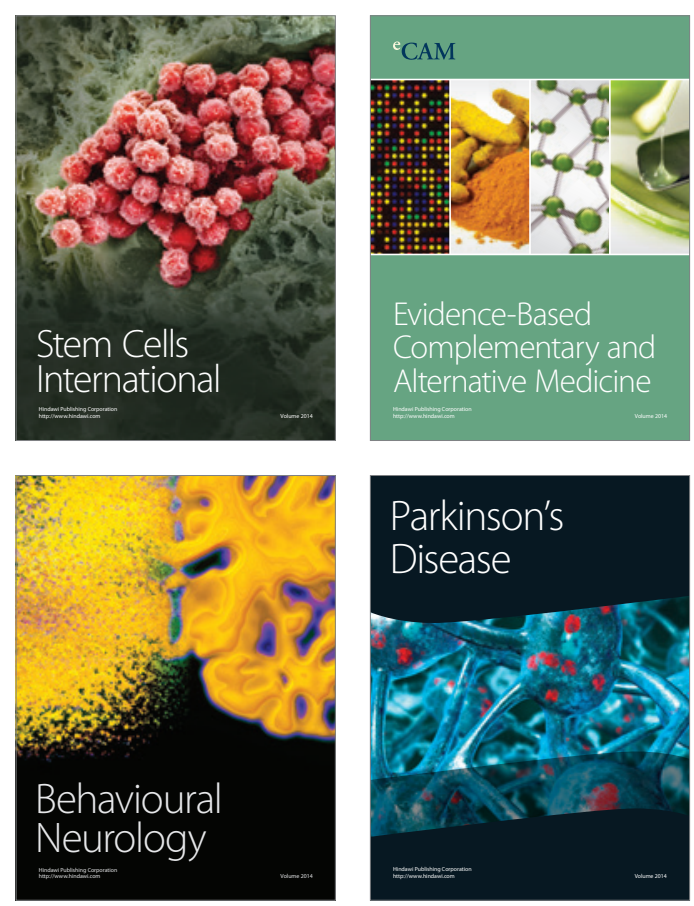

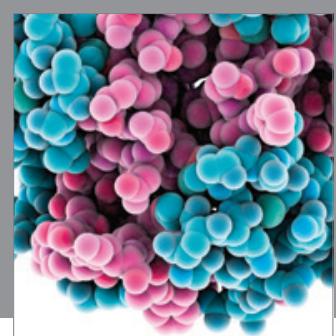

Journal of
Diabetes Research

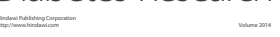

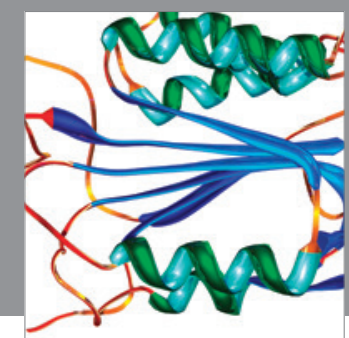

Disease Markers
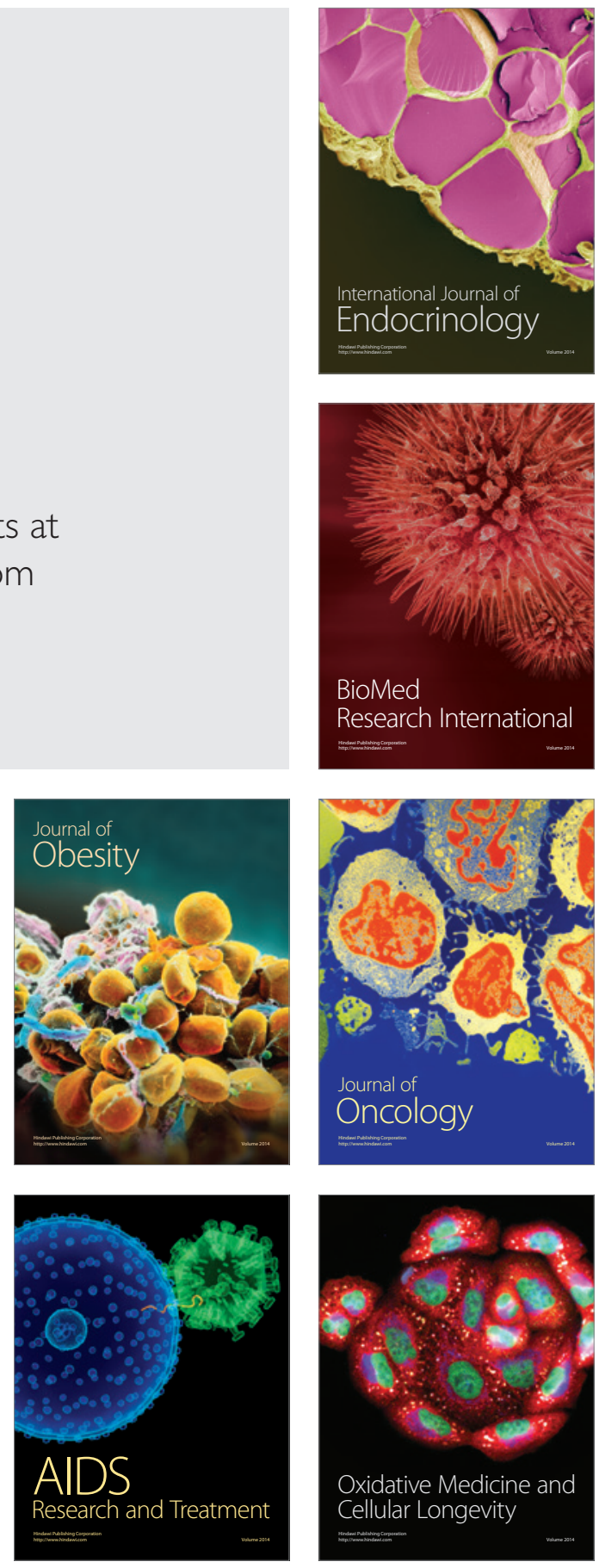